\title{
Wheezing caused by a Patent ductus arteriosus occluder:Case Report and Review of the Literature
}

\author{
Yong $\mathrm{Wu}^{1}$, Hanmin $\mathrm{Liu}^{2}$, Ling $\mathrm{Gu}^{2}, \mathrm{Li} \mathrm{Wang}^{3}$, and Ming $\mathrm{Su}^{4}$ \\ ${ }^{1}$ Mianyang Central Hospital \\ ${ }^{2}$ Sichuan University West China Second University Hospital \\ ${ }^{3}$ Chengdu Women's and Children's Central Hospital \\ ${ }^{4}$ Sichuan University West China Hospital
}

July 2, 2021

\begin{abstract}
Wheezing is often occurred in infants and young children with respiratory infections. For children with recurrent wheezing, after controlling their wheezing, they should be alert to rare diseases. Here, We report a case of wheezing following the application of the patent ductus arteriosus occlusion device ADOII (AGA Medical Corporation, Golden Valley,MN) with the occlusion device pressing against the inner diameter of the adjacent left main bronchus. After the pressure end of the occluder was removed, the child's wheezing was effectively relieved
\end{abstract}

\section{INTRODUCTION:}

Wheezing is a common symptom of respiratory infections in infants and young children, and about 30 percent of children develop wheezing from respiratory infections by the age of three【1】. In addition to infection, the Anatomic structure is abnormal, such as: Extrinsic to airway,Lymphadenopathy, Tumor,Diaphragmatic hernia,Vascular ring/aberrant vessel,Intrinsic to airway,Tracheomalaci, Foreign body, Endobronchial tuberculosis, Vocal cord dysfunction, Bronchopulmonary dysplasia,Congestive heart failure, Congenital lobar emphysema and Genetic/metabolic diseases also caused wheezing【2】.

Congenital heart disease is an important risk factor for infants hospitalized for wheezing【3】. Patent ductus arteriosus (PDA) is the third most common congenital heart disease in children[4]. Interventional therapy is an effective way to treat patent ductus arteriosus and can reduce the risk of hospitalization for pneumonia or wheezing in children with PDA 【5,6 】.

However, this paper reports a case of wheezing due to obstruction pressure on the airway after interventional treatment of patent ductus arteriosus. This has not been reported before, and a review of relevant literature was also carried out.

\section{CASE REPORT}

A 1-year and 3month old male child was hospitalized for cough and wheezing for half a month. Physical examination on admission: vital signs were stable, coarse and wet rales and a little wheezing could be heard in both lungs; physical examination of other systems was negative.

Past history: The children were hospitalized for "pneumonia" at the age of 20 days, 4 months, and 1 year. At 4 months of age, echocardiography revealed patent ductus arteriosus with a $2 \mathrm{~mm}$ wide left to right shunt and a large horizontal left to right shunt. At 11 months old, he underwent percutaneous patent ductus 
arteriosus occlusion under general anesthesia, AGA ADO II 3/4mm mushroom umbrella was used to seal the defect. No abnormal birth history, growth history and family history were found.

Blood routine, $\mathrm{C}$ reactive protein $(\mathrm{CRP})$ and blood biochemistry showed no abnormality. In sputum culture, a small amount of Streptococcus virifolia was found. Mycoplasma pneumoniae antibody IgM (-). Electrocardiogram was normal.

Cefoperazone and sulbactam were used for anti-infection treatment for 2 days, There was no significant improvement in symptoms.

Therefore, Further examination of the heart color ultrasound showed no shunt was seen in the ductus arteriosus after surgery (Figure .1).

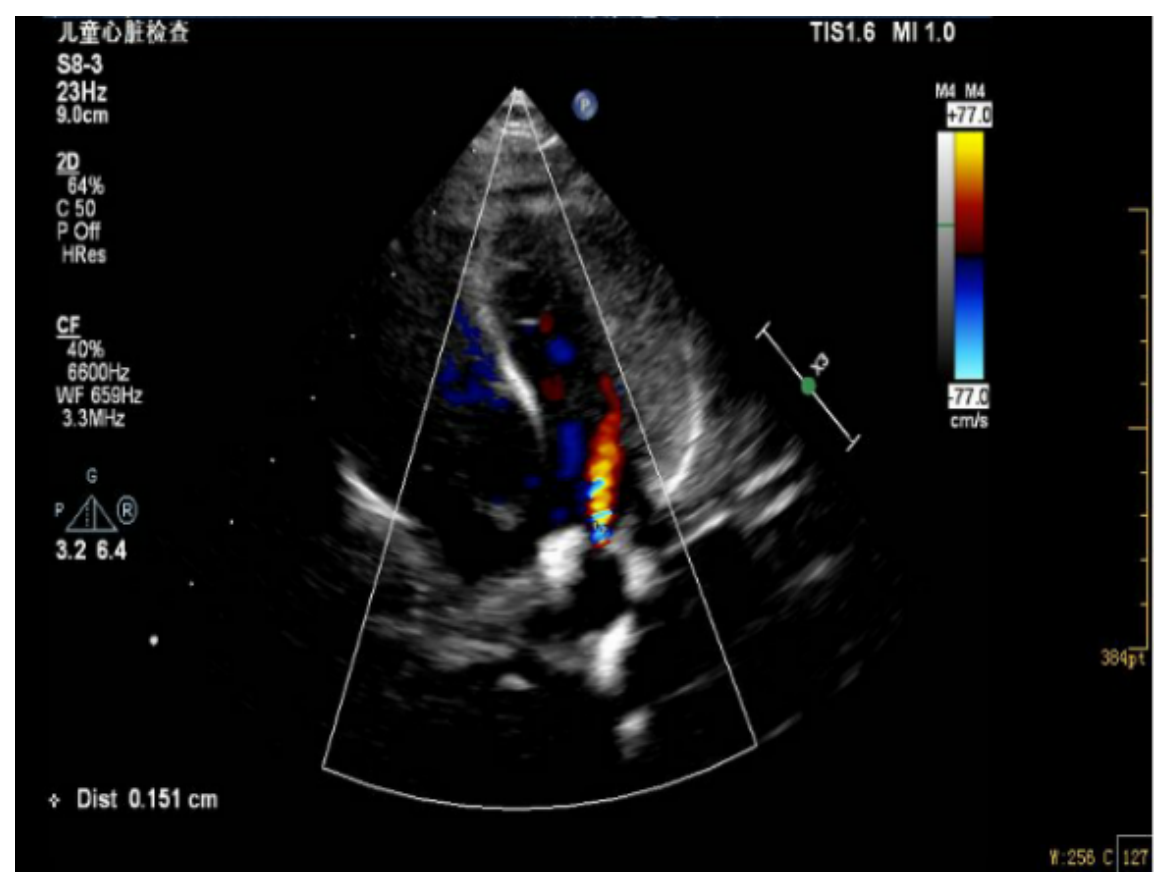




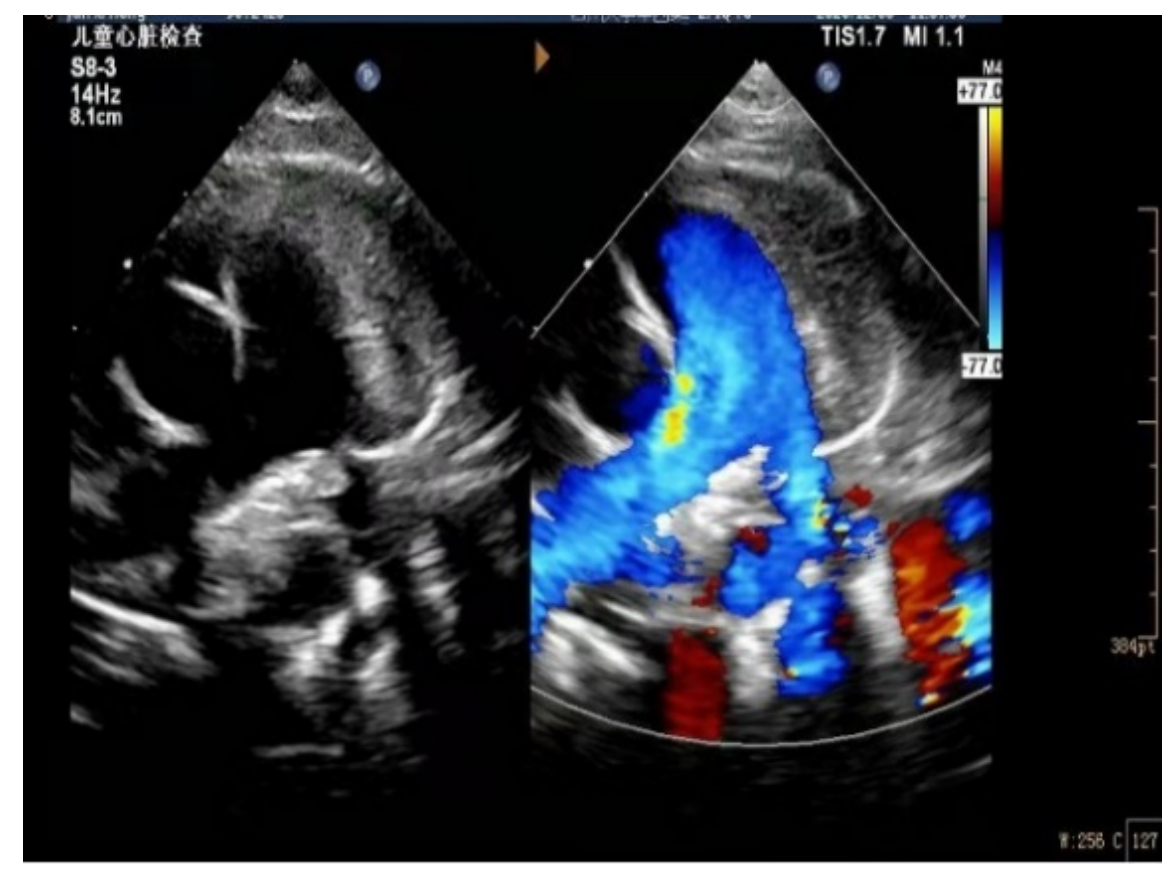

Chest CT scan showed flattening and stenosis in the middle segment of the left main bronchus immediately below the occlude (Figure .2). Fiberoptic bronchoscopy showed stenosis under external pressure of the left main bronchus.

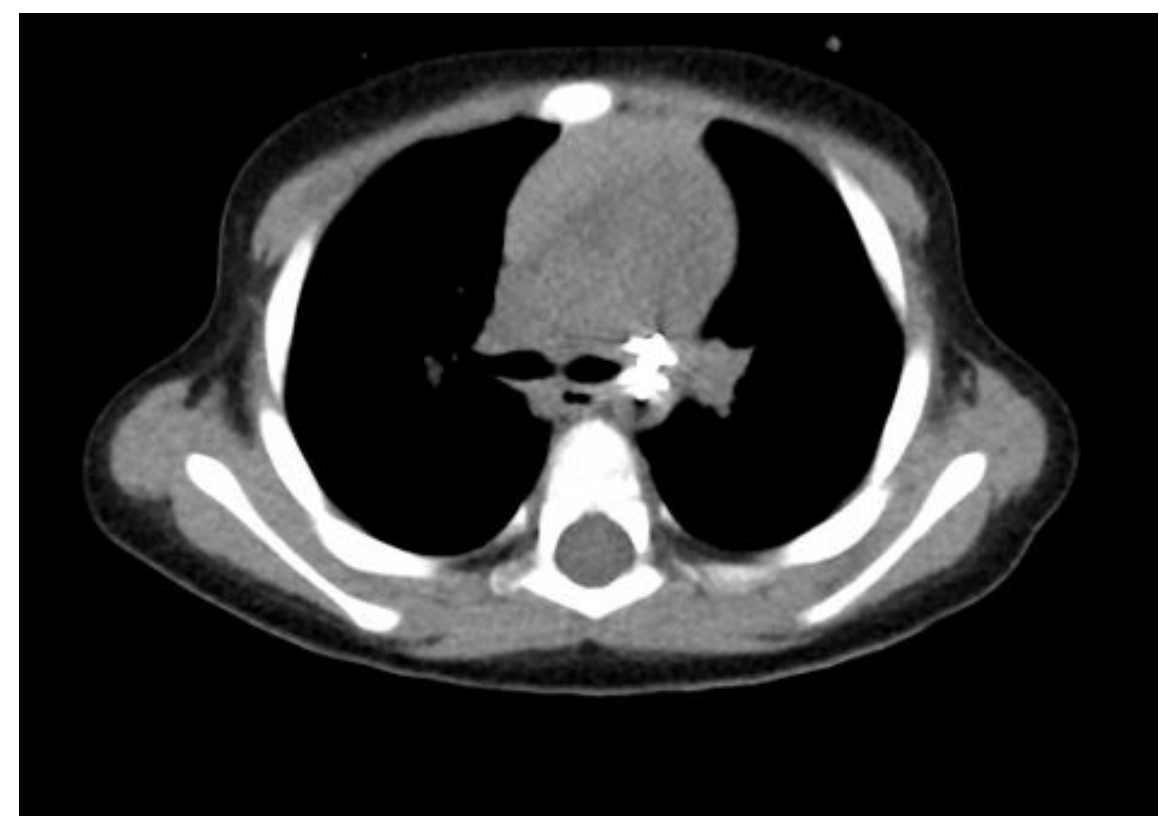




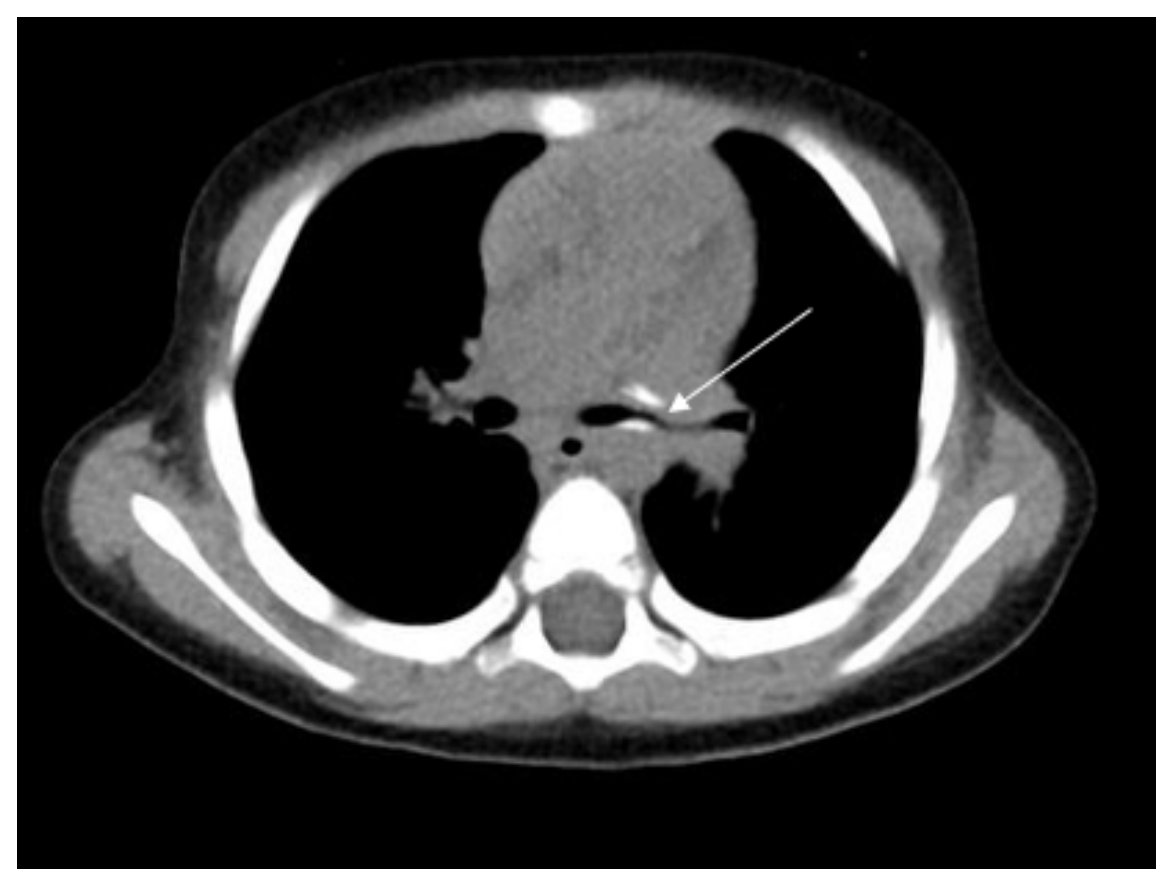

The patient was considered to have left main bronchial stenosis caused by PDA occlude. One week later, the child underwent arterial catheterization and intracardiac foreign body removal. The exploratory occluder could not be completely removed, so the pulmonary artery test occluding umbrella was cut off and removed, and the aortic side occluding umbrella was fixed in situ.

After the operation, the child's cough improved without wheezing and was discharged 3 days later. Chest CT reexamination 4 months later showed patency of the left main bronchus without compression (Figure $.3)$.

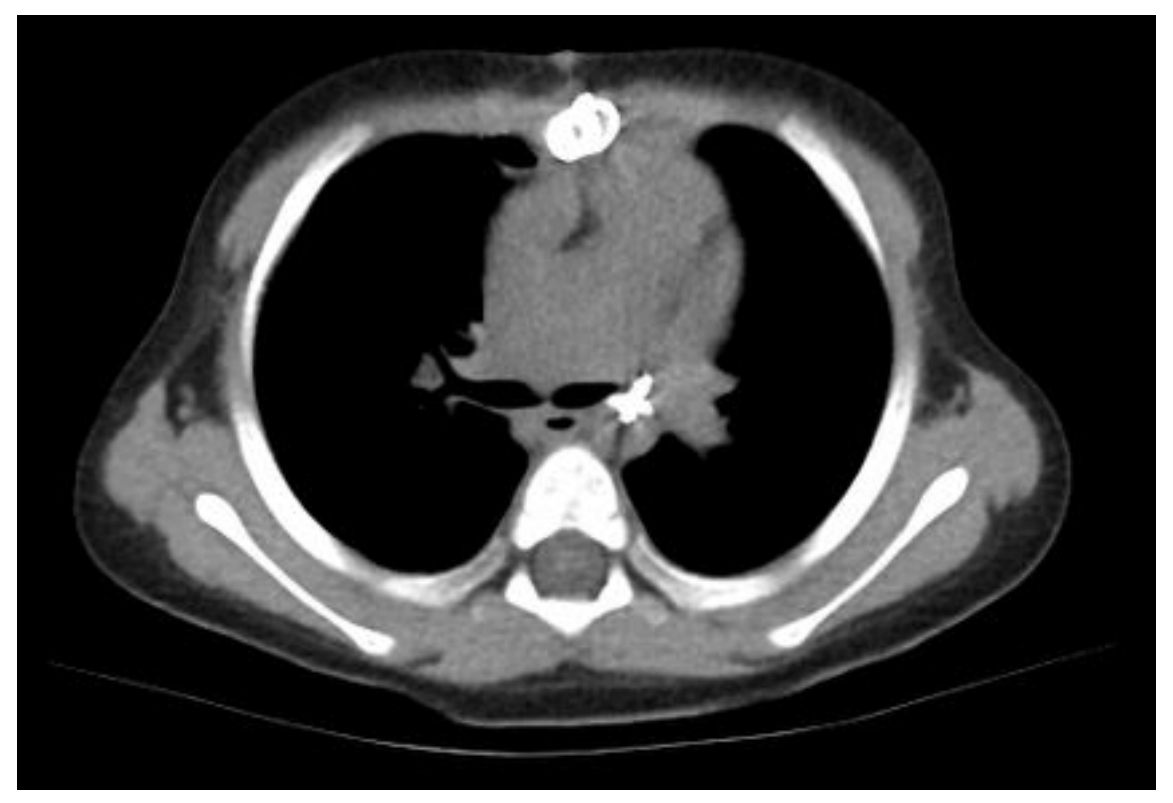




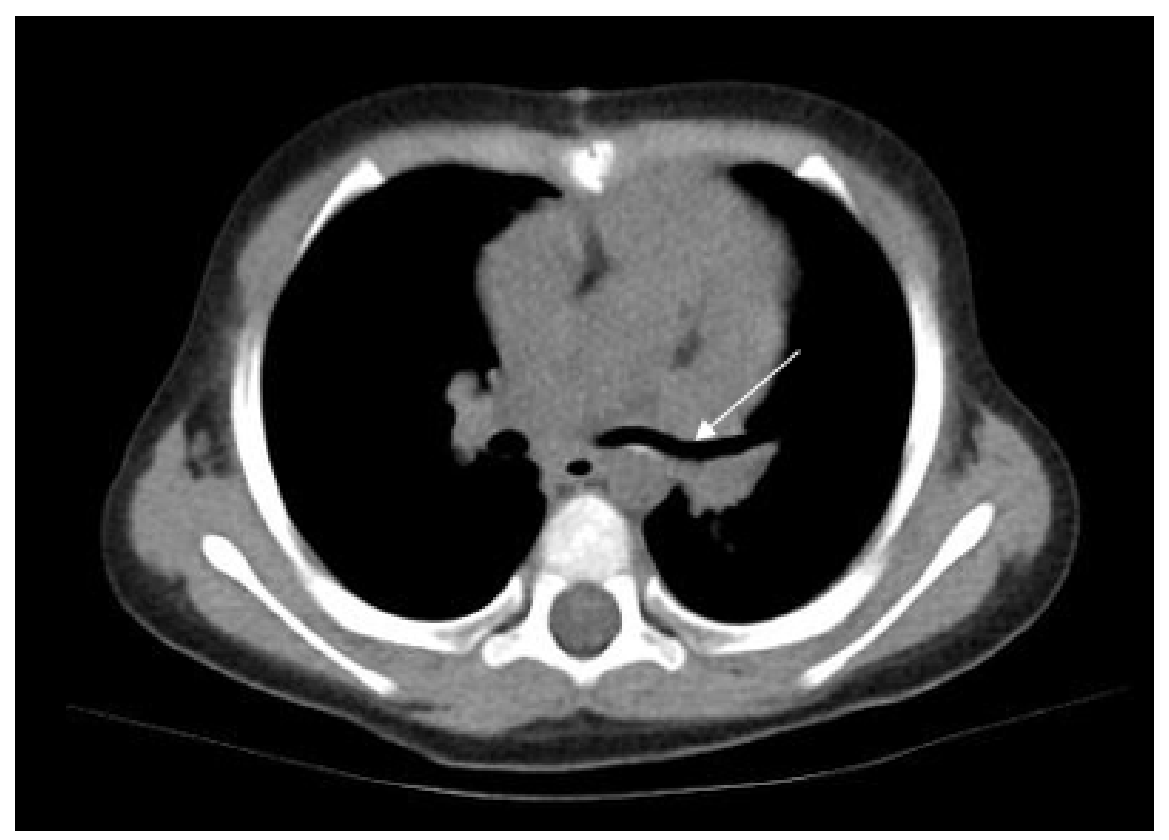

\section{DISCUSSION}

Wheezing has a high incidence of respiratory tract infection in infants and young children. In the UK, more than 70 per cent of babies and almost all children under the age of two become infected with bronchiolitis each year, Up to $20 \%$ of people with congenital heart disease are hospitalized and have a high morbidity and mortality rate[ 7]. Congenital heart disease is also considered a risk factor for recurrent wheezing [8] . Early intervention in children with preexisting heart disease can effectively reduce wheezing attacks caused by infection.

except to infection, compression of the tissues or blood vessels around the trachea can also cause wheezing, which can be the result of the abnormal relationship between the tracheobronchial tree and the vascular structure (the production of vascular rings), it may also be due to pulmonary artery expansion, left atrium enlargement, huge cardiac hypertrophy or external compression caused by intraluminal bronchial obstruction [9]. When patients have recurrent lower pulmonary infections and wheezing episodes, less common causes (including cardiac ones) should be considered, despite wheezing being controlled [10] .

The wheezing reported here was caused by compression of the left main bronchus following release of the ADOII occluder for patent ductus arteriosus.

For small PDAs, spring plugs were often used, the operation is complex, the residual shunt occurs higher. AGA (AGA Medical Corporation, Golden Valley, MN) ADOII has been in clinical use since 2008 and has solved this problem well. The new Amplatzer Catheter Occluder (ADO II) is safe and effective for PDA treatment in children up to 2 years of age, effectively closing PDAs under $2 \mathrm{~mm}[\mathbf{1 1 , 1 2}]$.

The ADOII was developed specifically for small to medium sized catheters[13], Major adverse events include obstruction of the descending aorta or pulmonary artery, but the overall incidence is low[14] .

Previous reports focused on the compression of blood vessels by embolization devices, but did not focus on the effect of blood vessels adjacent to the trachea. Therefore, we report these findings in order to evaluate the presence of adjacent tracheal compression in addition to the presence of intravascular flow obstruction during patent ductus arteriosus occlusion.

\section{CONCLUSION}


Here, for the first time, we report wheezing due to compression of the left main bronchus with a patent ductus arteriosus occlusion device.

After the application of occluders to occlude patent ductus arteriosus in the future, in addition to assessing the patency of blood flow in the vessels of the child, the patency of adjacent airways should also be evaluated to achieve a more complete assessment and reduce the incidence of related complications.

Compliance with ethical standards: The authors have read and confirmed their agreement according to the ICMJE authorship. The authors have confirmed that this article is not under consideration or published in any other publication

Conflict to interest: Nothing to report

\section{REFERENCE}

1. Piippo-Savolainen E, Korppi M. Wheezy babies-wheezy adults? Review on long-term outcome until adulthood after early childhood wheezing. Acta Paediatr. 2008 Jan;97(1):5-11.doi: 10.1111/j.16512227.2007.00558.x

2. Yehia M El-Gamal, Shereen S El-Sayed, Wheezing in infancy, World Allergy Organ J. 2011 May; 4(5): 85-90. doi: 10.1097/WOX.0b013e318216b41f

3. Minna Mecklin, Paula Heikkilä, Matti Korppi ,Low age, low birthweight and congenital heart disease are risk factors for intensive care in infants with bronchiolitis,Acta Paediatr, 2017 Dec;106(12): 2004-2010.doi: 10.1111/apa.14021

4. Benson LN, et al. paediatric cardiology, 2nd edition, London: Chuichil Livingstone,2002:1405-59

5. Yue Shu, Yilong Guo, Xiaoqi Wang, Dexing Zhou, Short-term results of interventional therapy for infants (7-36 months old) with patent ductus arteriosus and moderate-to-severe pulmonary hypertension: a retrospective study, Zhong hua Xin Xue Guan Bing Za Zhi. 2012 Apr;40(4):283-8. PMID: 22801304

6. Zhi-Yuan Song, Mao-Qin Shu, Hou-Yuan Hu,et al, Clinical efficiency and safety analysis of transcatheter interventional therapy for compound congenital cardiovascular abnormalities, Clin Cardiol. 2007 Oct;30(10):518-21. doi: 10.1002/clc.20149

7. Robert M R Tulloh, Sarah Bury,Prevention and prophylaxis of respiratory syncytial virus in pediatric cardiology: a UK perspective, Future Cardiol. 2014 Mar;10(2):235-42. doi: 10.2217/fca.14.4

8. Abdulkadir Bozaykut, Ahu Paketci, Rabia Gonul Sezer, Cem Paketci, Evaluation of risk factors for recurrent wheezing episodes, J Clin Med Res. 2013 Oct;5(5):395-400. doi: 10.4021/jocmr1543w

9. Barry D Kussman, Tal Geva, Francis X McGowan,Cardiovascular causes of airway compression,Paediatr Anaesth.2004 Jan;14(1):60-74. doi: 10.1046/j.1460-9592.2003.01192.x

10. A Pisanti, R Vitiello, Wheezing as the sole clinical manifestation of cor triatriatum,Pediatr Pulmonol.2000 Oct;30(4):346-9.doi:10.1002/1099-0496(200010)30:4<346:: aid-ppul12>3.0.co;2-p

11. Basil Thanopoulos, Nikolaos Eleftherakis, Konstantinos Tzannos, Christodoulos Stefanadis, Transcatheter closure of the patent ductus arteriosus using the new Amplatzer duct occluder: initial clinical applications in children,Am Heart J. 2008 Nov;156(5): 917.e1-917.e6. doi: 10.1016/j.ahj.2008.08.001

12. Rasha I Ammar, Ranya A Hegazy,Percutaneous closure of medium and large PDAs using amplatzer duct occluder (ADO) I and II in infants: safety and efficacy,J Invasive Cardiol. 2012 Nov;24(11):579-82. PMID: 23117312

13. Gruenstein DH, Ebeid M, Radtke W, Moore P, Holzer R, Justino H. Transcatheter closure of patent ductus arteriosus using the AMPLATZER duct occluder II (ADO II). Catheter Cardiovasc Interv. 2017 May;89(6):1118-1128. doi: 10.1002/ccd.26968 
14. Ji, W; Li, F; Gao, W; Chen, YW, et al. transcatheter closure of patent ductus arteriosus with the new Amplatzer Duct Occluder II, Zhonghua Er Ke Za Zhi.2013 Feb ;51(2) :126-9, PMID: 23527979 